\title{
On compact hyperbolic manifolds of Euler characteristic two
}

\author{
VINCENT EMERY
}

\begin{abstract}
We prove that for $n>4$ there is no compact arithmetic hyperbolic $n$-manifold whose Euler characteristic has absolute value equal to 2. In particular, this shows the nonexistence of arithmetically defined hyperbolic rational homology $n$-spheres with $n$ even and different than 4 .
\end{abstract}

22E40; 55C 35, 51M25

Dedicated to the memory of Colin Maclachlan

\section{Main result and discussion}

\subsection{Smallest hyperbolic manifolds}

Let $\mathbb{H}^{n}$ be the hyperbolic $n$-space. By a hyperbolic $n$-manifold we mean an orientable manifold $M=\Gamma \backslash \mathbb{H}^{n}$, where $\Gamma$ is a torsion free discrete subgroup $\Gamma \subset \operatorname{Isom}^{+}\left(\mathbb{H}^{n}\right)$. The set of volumes of hyperbolic $n$-manifolds being well ordered, it is natural to try to determine for each dimension $n$ the hyperbolic manifolds of smallest volume. For $n=3$ this problem has recently been solved by Gabai, Meyerhoff and Milley in [15], the smallest volume being achieved by a unique compact manifold, the Weeks manifold. When $n$ is even the volume is proportional to the Euler characteristic, and this allows us to formulate the problem in terms of finding the hyperbolic manifolds $M$ with smallest $|\chi(M)|$. In particular this observation solves the problem in the case of surfaces. For $n>3$, noncompact hyperbolic $n$-manifolds $M$ with $|\chi(M)|=1$ have been found for $n=4,6$; see Everitt, Ratcliffe and Tschantz [13].

In the present paper we consider the case of compact manifolds of even dimension. In particular, such manifolds have even Euler characteristic (see Kellerhals and Zehrt [17, Theorem 1.2]). We restrict ourselves to the case of arithmetic manifolds, where Prasad's formula [20] can be used to study volumes. We complete the proof of the following result.

Theorem 1 Let $n>5$. There is no compact arithmetic manifold $M=\Gamma \backslash \mathbb{H}^{n}$ with $|\chi(M)|=2$. 
The result for $n>10$ already follows from the work of Belolipetsky [2; 3], also based on Prasad's volume formula. More precisely, Belolipetsky determined the smallest Euler characteristic $|\chi(\Gamma)|$ for arithmetic orbifold quotients $\Gamma \backslash \mathbb{H}^{n}$ ( $n$ even). This smallest value grows fast with the dimension $n$, and for compact quotients we have $|\chi(\Gamma)|>2$ for $n>10$. That the result of nonexistence holds for $n$ high enough is already a consequence of Borel and Prasad's general finiteness result [7], which was the first application of Prasad's formula. The proof of Theorem 1 for $n=6,8,10$ requires a more precise analysis of the Euler characteristic of arithmetic subgroups $\Gamma \subset \mathrm{PO}(n, 1)$, and in particular of the special values of Dedekind zeta functions that appear as factors of $\chi(\Gamma)$.

For $n=4$, the corresponding problem is not solved, but there is the following result [3].

Theorem 2 (Belolipetsky) If $M=\Gamma \backslash \mathbb{H}^{4}$ is a compact arithmetic manifold with $\chi(M) \leq 16$, then $\Gamma$ arises as a (torsion free) subgroup of the following hyperbolic Coxeter group:

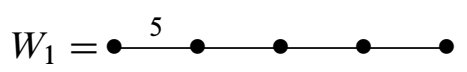

An arithmetic (orientable) hyperbolic 4-manifold of Euler characteristic 16 was first constructed by Conder and Maclachlan in [11], using the presentation of $W_{1}$ to obtain a torsion free subgroup with the help of a computer. Further examples with $\chi(M)=16$ have been obtained by Long in [18] by considering a homomorphism from $W_{1}$ onto the finite simple group $\mathrm{PSp}_{4}(4)$.

\subsection{Hyperbolic homology spheres}

Our original motivation for Theorem 1 was the problem of existence of hyperbolic homology spheres. A homology $n$-sphere (resp. rational homology $n$-sphere) is a $n$-manifold $M$ that possesses the same integral (resp. rational) homology as the $n$-sphere $S^{n}$. This forces $M$ to be compact and orientable.

Rational homology $n$-spheres $M$ have $\chi(M)=2$ if $n$ is even. On the other hand, for $M=\Gamma \backslash \mathbb{H}^{n}$ with $n=4 k+2$ we have $\chi(M)<0$ (cf Serre [25, Proposition 23]), and this excludes the possibility of hyperbolic rational homology spheres for those dimensions. For $n$ even, Wang's finiteness theorem [28] implies that there is only a finite number of hyperbolic rational homology $n$-spheres. Theorem 1 shows the nonexistence of arithmetic rational homology spheres for $n>5$ even. 
For odd dimensions, $\chi(M)=0$ and a priori the volume is not a limitation for the existence of hyperbolic (rational) homology spheres. In fact, an infinite tower of covers by hyperbolic integral homology 3-spheres has been constructed by Baker, Boileau and Wang in [1]. In [8] Calegari and Dunfield constructed an infinite tower of hyperbolic rational homology 3-spheres that are arithmetic and obtained by congruence subgroups. Note that a recent conjecture of Bergeron and Venkatesh predicts a lot of torsion in the homology groups of such a "congruence tower" of arithmetic $n$-manifolds with $n$ odd [5].

\subsection{Locally symmetric homology spheres}

Instead of considering hyperbolic homology spheres, one can more generally look for homology spheres that are locally isometric to a given symmetric space of nonpositive nonflat sectional curvature. Such a symmetric space $X$ is said to be of noncompact type, and it is classical that $X$ can be written as $G / K$, where $G$ is a connected real semisimple Lie group with trivial center with $K \subset G$ a maximal compact subgroup. Moreover, $G$ identifies as a finite index subgroup in the group of isometries of $X$ (of index two if $G$ is simple).

Let us explain why the case $X=\mathbb{H}^{n}$ is the main source of locally symmetric rational homology spheres (among $X$ of noncompact type). Let $M$ be a compact orientable manifold locally isometric to $X$. Then $M$ can be written as $\Gamma \backslash X$, where $\Gamma \cong \pi_{1}(M)$ is a discrete subgroup of isometries of $X$. We will suppose that $\Gamma \subset G$, for $G$ as above. Let $X_{u}$ be the compact dual of $X$. We have the following general result (see Borel [6, Sections 3.2 and 10.2]).

Proposition 3 There is an injective homomorphism $H^{j}\left(X_{u}, \mathbb{C}\right) \rightarrow H^{j}(\Gamma \backslash X, \mathbb{C})$, for each $j$.

In particular, if $\Gamma \backslash X$ is a rational homology sphere, then so is $X_{u}$. Note that the compact dual of $X=\mathbb{H}^{n}$ is the genuine sphere $S^{n}$. By looking at the classification of compact symmetric spaces, Johnson showed the following in [16, Theorem 7].

Corollary 4 If $M=\Gamma \backslash X$ is a rational homology $n$-sphere with $\Gamma \subset G$, then $X$ is either the hyperbolic $n$-space $\mathbb{H}^{n}$ (with $n \neq 4 k+2$ ), or $X=\operatorname{PSL}_{3}(\mathbb{R}) / \operatorname{PSO}(3)$ (which has dimension 5).

Proposition 3 shows that the correct problem to look at - rather than homology spheres - is the existence of locally symmetric spaces $\Gamma \backslash X$ with the same (rational) homology as the compact dual $X_{u}$. When $X$ is the complex hyperbolic plane $\mathbb{H}_{\mathbb{C}}^{2}$, the 
compact dual is the projective plane $\mathbb{P}_{\mathbb{C}}^{2}$, and the quotients $\Gamma \backslash X$ are compact complex surfaces called fake projective planes. Their classification was recently obtained by the work of Prasad and Yeung [21], together with Cartwright and Steger [9] who performed the necessary computer search. Later, Prasad and Yeung also considered the problem of the existence of more general arithmetic fake Hermitian spaces [22; 23].

The present paper uses the same methodology as in Prasad and Yeung's work, the main ingredient being the volume formula.

Acknowledgements It is a pleasure to thank Gopal Prasad, who suggested this research project. This work was supported by the Swiss National Science Foundation, project number PA00P2-139672.

\section{Proof of Theorem 1}

Let $G=\mathrm{PO}(n, 1)^{\circ} \cong \operatorname{Isom}^{+}\left(\mathbb{H}^{n}\right)$ and consider the universal covering $\phi: \operatorname{Spin}(n, 1) \rightarrow G$. For our purpose it will be easier to work with lattices in $\operatorname{Spin}(n, 1)$. A lattice $\bar{\Gamma} \subset G$ is arithmetic exactly when $\Gamma=\phi^{-1}(\bar{\Gamma})$ is an arithmetic subgroup of $\operatorname{Spin}(n, 1)$. Since the covering $\phi$ is twofold, we have $\chi(\Gamma)=\frac{1}{2} \chi(\bar{\Gamma})$, where $\chi$ is the Euler characteristic in the sense of Wall. In particular, if $M=\bar{\Gamma} \backslash \mathbb{H}^{n}$ is a manifold with $|\chi(M)|=2$, then $|\chi(\Gamma)|=1$. Thus, Theorem 1 is an obvious consequence of the following proposition. The proof relies on the description of arithmetic subgroups with the help of Bruhat-Tits theory, as done for instance in $[7 ; 20]$. An introduction can be found in the author's work [12]. We also refer to [27] for the needed facts from Bruhat-Tits theory.

Proposition 5 Let $n>4$. There is no cocompact arithmetic lattice $\Gamma \subset \operatorname{Spin}(n, 1)$ such that $\chi(\Gamma)$ is a reciprocal integer, ie, such that $\chi(\Gamma)=1 / q$ for some $q \in \mathbb{Z}$.

Proof We can assume that $n$ is even. Let $\Gamma \subset \operatorname{Spin}(n, 1)$ be a cocompact lattice. Clearly, it suffices to prove the proposition for $\Gamma$ maximal. In this case, $\Gamma$ can be written as the normalizer $\Gamma=N_{\operatorname{Spin}(n, 1)}(\Lambda)$ of some principal arithmetic subgroup $\Lambda$ (see [7, Proposition 1.4]). By definition, there exists a number field $k \subset \mathbb{R}$ and a $k$-group $\boldsymbol{G}$ with $\boldsymbol{G}(\mathbb{R}) \cong \operatorname{Spin}(n, 1)$ such that $\Lambda=\boldsymbol{G}(k) \cap \prod_{v \in V_{\mathrm{f}}} P_{v}$, for some coherent collection $\left(P_{v}\right)_{v \in V_{\mathrm{f}}}$ of parahoric subgroups $P_{v} \subset \boldsymbol{G}\left(k_{v}\right)$ (indexed by the set $V_{\mathrm{f}}$ of finite places of $k$ ). It follows from the classification of algebraic groups (cf Tits [26]) that $\boldsymbol{G}$ is of type $\mathrm{B}_{r}$ with $r=n / 2(>2)$, the field $k$ is totally real, and (using Godement's criterion) $k \neq \mathbb{Q}$. Let us denote by $d$ the degree $[k: \mathbb{Q}]$. 
Let $T \subset V_{\mathrm{f}}$ be the set of places where $P_{v}$ is not hyperspecial. By Prasad's volume formula (see [20] and [7, Section 4.2]), we have

$$
|\chi(\Lambda)|=2\left|D_{k}\right|^{r^{2}+r / 2} C(r)^{d} \prod_{j=1}^{r} \zeta_{k}(2 j) \prod_{v \in T} \lambda_{v},
$$

with $D_{k}$ (resp. $\zeta_{k}$ ) the discriminant (resp. Dedekind zeta function) of $k$; the constant $C(r)$ is given by

$$
C(r)=\prod_{j=1}^{r} \frac{(2 j-1) !}{(2 \pi)^{2 j}},
$$

and each $\lambda_{v}$ is given by the formula

$$
\lambda_{v}=\frac{1}{\left(q_{v}\right)^{\left(\operatorname{dim} \mathcal{M}_{v}-\operatorname{dim} \boldsymbol{M}_{v}\right) / 2}} \frac{\left|\mathcal{M}\left(\mathfrak{f}_{v}\right)\right|}{\left|\boldsymbol{M}_{v}\left(\mathfrak{f}_{v}\right)\right|},
$$

where $\mathfrak{f}_{v}$ is the residue field of $k_{v}$, of size $q_{v}$, and the reductive $\mathfrak{f}_{v}$-groups $\boldsymbol{M}_{v}$ and $\mathcal{M}_{v}$ associated with $P_{v}$ are those described in [20]. By definition $\mathcal{M}_{v}$ is semisimple of type $\mathrm{B}_{r}$.

\begin{tabular}{l|l|c}
$\boldsymbol{G} / k_{v}$ & isogeny type of $\boldsymbol{M}_{v}$ & $\lambda_{v}$ \\
\hline split & $\mathrm{B}_{r-1} \times\left(\right.$ split $\left.\mathrm{GL}_{1}\right)$ & $\frac{q^{2 r}-1}{q-1}$ \\
& $\mathrm{D}_{i} \times \mathrm{B}_{r-i}(i=2, \ldots, r-1)$ & $\frac{\left(q^{i}+1\right) \prod_{k=i+1}^{r}\left(q^{2 k}-1\right)}{\prod_{k=1}^{r-i}\left(q^{2 k}-1\right)}$ \\
& ${ }^{1} \mathrm{D}_{r}$ & $q^{r}+1$ \\
nonsplit & $\mathrm{B}_{r-1} \times\left(\right.$ nonsplit $\left.\mathrm{GL}_{1}\right)$ & $\frac{q^{2 r}-1}{q+1}$ \\
& ${ }^{2} \mathrm{D}_{i+1} \times \mathrm{B}_{r-i-1}(i=1, \ldots, r-2)$ & $\frac{\left(q^{i+1}-1\right) \prod_{k=i+2}^{r}\left(q^{2 k}-1\right)}{\prod_{k=1}^{r-i-1}\left(q^{2 k}-1\right)}$ \\
& ${ }^{2} \mathrm{D}_{r}$ & $q^{r}-1$
\end{tabular}

Table 1: $\lambda_{v}$ for $P_{v}$ of maximal type

A necessary condition for $\Gamma=N_{\boldsymbol{G}(\mathbb{R})}(\Lambda)$ to be maximal is that each $P_{v}$ defining $\Lambda$ has maximal type in the sense of Ryzhkov and Chernousov [24]. We list in Table 1 the factors $\lambda_{v}$ corresponding to parahoric subgroups $P_{v}$ of maximal types (to improve the readability we set $q_{v}=q$ in the formulas). This list of maximal type and the formulas for $\lambda_{v}$ are essentially the same as in [2, Table 1]: the only difference is a factor of 2 in the denominator of some $\lambda_{v}$, which can be explained from the fact that Belolipetsky did not work with $\boldsymbol{G}$ simply connected. 
From [7, Section 5] (cf also [12, Chapter 12]) we can deduce that the index $[\Gamma: \Lambda]$ of $\Lambda$ in its normalizer has the following property:

$$
[\Gamma: \Lambda] \text { divides } h_{k} 2^{d} 4^{\# T}
$$

Moreover, a case by case analysis of the possible factor $\lambda_{v}$ shows that $\lambda_{v}>4$, so that $4^{-\# T} \prod_{v \in T} \lambda_{v} \geq 1$ (with equality exactly when $T$ is empty). We thus have the following lower bound for the Euler characteristic of any maximal arithmetic subgroup $\Gamma \subset \operatorname{Spin}(n, 1)$ :

$$
|\chi(\Gamma)| \geq \frac{2}{h_{k}}\left(\frac{C(r)}{2}\right)^{d}\left|D_{k}\right|^{r^{2}+r / 2} \zeta_{k}(2) \cdots \zeta_{k}(2 r)
$$

We make use of the following upper bound for the class number (see for instance Belolipetsky and the author [4, Section 7.2]):

$$
h_{k} \leq 16\left(\frac{\pi}{12}\right)^{d}\left|D_{k}\right|
$$

which together with the basic inequality $\zeta_{k}(2 j)>1$ transforms (6) into

$$
|\chi(\Gamma)|>\frac{1}{8}\left(\frac{6 \cdot C(r)}{\pi}\right)^{d}\left|D_{k}\right|^{r^{2}+r / 2-1} .
$$

Moreover, according to Odlyzko [19, Table 4], we have that for a degree $d \geq 5$ the discriminant of $k$ is larger than $(6.5)^{d}$. With this estimates we can check that for $r \geq 3$ and $d \geq 5$ we have $|\chi(\Gamma)|>1$. For the lower degrees, if we suppose that $|\chi(\Gamma)| \leq 1$, we obtain upper bounds for $\left|D_{k}\right|$ from Equation (8). This upper bounds exclude the existence of such a $\Gamma$ for $r \geq 6$ (which is already clear from the work of Belolipetsky [2]). For $r=3$ (where the bounds are the worst) we obtain:

$$
\begin{aligned}
& d=2:\left|D_{k}\right| \leq 28 \\
& d=3:\left|D_{k}\right| \leq 134 \\
& d=4:\left|D_{k}\right| \leq 640
\end{aligned}
$$

From the existing tables of number fields (eg, $[10 ; 14])$ we can list the possibilities this leaves us for $k$. We find that no field with $d=4$ can appear, and for $d=2,3$ all possibilities have class number $h_{k}=1$. Using Equation (7) with $h_{k}=1$ we then improve the upper bounds for $\left|D_{k}\right|$ and thus shorten the list of possible fields. For $r=5$ only $\left|D_{k}\right|=5$ arises, and for $r=4$ we have $\left|D_{k}\right| \leq 11$ (the possibility $d=3$ is excluded here). For $r=3$, we are left with $\left|D_{k}\right| \leq 20$ when $d=2$, and $\left|D_{k}\right|=49$ or 81 when $d=3$. 
With $h_{k}=1$, using the functional equation for $\zeta_{k}$ and the property (5) for the index $[\Gamma: \Lambda]$, we can express the Euler characteristic of $\Gamma$ as

$$
|\chi(\Gamma)|=\frac{1}{2^{a}} \prod_{v \in T} \lambda_{v} \prod_{j=1}^{r}\left|\zeta_{k}(1-2 j)\right|
$$

for some integer $a$. The special values $\zeta_{k}(1-2 j)$, which are rational by the KlingenSiegel theorem, can be computed with the software Pari/GP (cf Remark 6). We list in Table 2 the values we need. We check that for every field $k$ under consideration a prime factor greater than 2 appear in the numerator of the product $\prod_{j=1}^{r}\left|\zeta_{k}(1-2 j)\right|$. A direct computation for $r=3,4,5$ shows that the formula in Table 1 for each factor $\lambda_{v}$ is actually given by a polynomial in $q$ (this seems to hold for any $r$ ). In particular, we always have $\lambda_{v} \in \mathbb{Z}$, and we conclude from (9) that $|\chi(\Gamma)|$ cannot be a reciprocal integer.

\begin{tabular}{c|c|c|c|c|c|c} 
degree & $\left|D_{k}\right|$ & $\zeta_{k}(-1)$ & $\zeta_{k}(-3)$ & $\zeta_{k}(-5)$ & $\zeta_{k}(-7)$ & $\zeta_{k}(-9)$ \\
\hline$d=2$ & 5 & $1 / 30$ & $1 / 60$ & $67 / 630$ & $361 / 120$ & $412751 / 1650$ \\
& 8 & $1 / 12$ & $11 / 120$ & $361 / 252$ & $24611 / 240$ & \\
& 12 & $1 / 6$ & $23 / 60$ & $1681 / 126$ & & \\
& 13 & $1 / 6$ & $29 / 60$ & $33463 / 1638$ & & \\
& 17 & $1 / 3$ & $41 / 30$ & $5791 / 63$ & & \\
& 49 & $-1 / 21$ & $79 / 210$ & $-7393 / 63$ & &
\end{tabular}

Table 2: Special values of $\zeta_{k}$

This completes the proof.

Remark 6 The function zetak in Pari/GP allows us to obtain approximate values for $\zeta_{k}(1-2 j)$. On the other hand the size of the denominator of the product $\prod_{j=1}^{m} \zeta_{k}(1-2 j)$ can be bounded by the method described in [25, Section 3.7]. By recursion on $m$, this allows to ascertain that the values $\zeta_{k}(1-2 j)$ correspond exactly to the fractions given in Table 2.

Remark 7 The fact that for $\left|D_{k}\right|=5$ the value $\zeta_{k}(-1) \zeta_{k}(-3)$ has trivial numerator explains why the proof fails for $n=4$ (ie, $r=2$ ). And indeed there is a principal arithmetic subgroup $\Gamma \subset \operatorname{Spin}(4,1)$ with $|\chi(\Gamma)|=\frac{1}{14400}$ and whose image in $\operatorname{Isom}^{+}\left(\mathbb{H}^{4}\right)$ is contained as an index- 2 subgroup of the Coxeter group $W_{1}$. On the other hand, for 
$\left|D_{k}\right|>5$ the appearance of a nontrivial numerator in $\zeta_{k}(-3)$ shows - at least for the fields considered in Table 2 - the impossibility of a $\Gamma$ defined over $k$ with $\chi(\Gamma)$ a reciprocal integer. This is the first step in Belolipetsky's proof of Theorem 2.

\section{References}

[1] M Baker, M Boileau, S Wang, Towers of covers of hyperbolic 3-manifolds, Rend. Istit. Mat. Univ. Trieste 32 (2001) 35-43 MR1893391 Dedicated to the memory of Marco Reni

[2] M Belolipetsky, On volumes of arithmetic quotients of $\mathrm{SO}(1, n)$, Ann. Sc. Norm. Super. Pisa Cl. Sci. 3 (2004) 749-770 MR2124587

[3] M Belolipetsky, Addendum to: “On volumes of arithmetic quotients of $\operatorname{SO}(1, n)$ ", Ann. Sc. Norm. Super. Pisa Cl. Sci. 6 (2007) 263-268 MR2352518

[4] M Belolipetsky, V Emery, On volumes of arithmetic quotients of $\mathrm{PO}(n, 1)^{\circ}, n$ odd, Proc. Lond. Math. Soc. 105 (2012) 541-570 MR2974199

[5] N Bergeron, A Venkatesh, The asymptotic growth of torsion homology for arithmetic groups, J. Inst. Math. Jussieu 12 (2013) 391-447 MR3028790

[6] A Borel, Stable real cohomology of arithmetic groups, Ann. Sci. École Norm. Sup. 7 (1974) 235-272 MR0387496

[7] A Borel, G Prasad, Finiteness theorems for discrete subgroups of bounded covolume in semi-simple groups, Inst. Hautes Études Sci. Publ. Math. (1989) 119-171 MR1019963

[8] F Calegari, NM Dunfield, Automorphic forms and rational homology 3-spheres, Geom. Topol. 10 (2006) 295-329 MR2224458

[9] D I Cartwright, T Steger, Enumeration of the 50 fake projective planes, C. R. Math. Acad. Sci. Paris 348 (2010) 11-13 MR2586735

[10] H Cohen, et al., The Bordeaux database Available at megrez.math.u-bordeaux.fr

[11] M Conder, C Maclachlan, Compact hyperbolic 4-manifolds of small volume, Proc. Amer. Math. Soc. 133 (2005) 2469-2476 MR2138890

[12] V Emery, Du volume des quotients arithmétiques de l'espace hyperbolique, $\mathrm{PhD}$ thesis, University of Fribourg (2009) Available at http://homeweb1.unifr.ch/ kellerha/pub/DissVEmery-final-09.pdf

[13] B Everitt, J G Ratcliffe, S T Tschantz, Right-angled Coxeter polytopes, hyperbolic six-manifolds, and a problem of Siegel, Math. Ann. 354 (2012) 871-905 MR2983072

[14] S Freundt, QaoS online database Available at http://qaos.math.tu-berlin.de

[15] D Gabai, R Meyerhoff, P Milley, Minimum volume cusped hyperbolic three-manifolds, J. Amer. Math. Soc. 22 (2009) 1157-1215 MR2525782 
[16] F E A Johnson, Locally symmetric homology spheres and an application of Matsushima's formula, Math. Proc. Cambridge Philos. Soc. 91 (1982) 459-466 MR654091

[17] R Kellerhals, T Zehrt, The Gauss-Bonnet formula for hyperbolic manifolds of finite volume, Geom. Dedicata 84 (2001) 49-62 MR1825344

[18] C Long, Small volume closed hyperbolic 4-manifolds, Bull. Lond. Math. Soc. 40 (2008) 913-916 MR2439657

[19] A M Odlyzko, Bounds for discriminants and related estimates for class numbers, regulators and zeros of zeta functions: A survey of recent results, Sém. Théor. Nombres Bordeaux 2 (1990) 119-141 MR1061762

[20] G Prasad, Volumes of $S$-arithmetic quotients of semi-simple groups, Inst. Hautes Études Sci. Publ. Math. (1989) 91-117 MR1019962 With an appendix by Moshe Jarden and the author

[21] G Prasad, S-K Yeung, Fake projective planes, Invent. Math. 168 (2007) 321-370 MR2289867

[22] G Prasad, S-K Yeung, Arithmetic fake projective spaces and arithmetic fake Grassmannians, Amer. J. Math. 131 (2009) 379-407 MR2503987

[23] G Prasad, S-K Yeung, Nonexistence of arithmetic fake compact Hermitian symmetric spaces of type other than $A_{n}(n \leq 4)$, J. Math. Soc. Japan 64 (2012) 683-731 MR2965425

[24] A A Ryzhkov, V I Chernousov, On the classification of maximal arithmetic subgroups of simply connected groups, Mat. Sb. 188 (1997) 127-156 MR1481667 In Russian; translated in Mat. Sb. 188 (1997) 1385-1413

[25] J-P Serre, Cohomologie des groupes discrets, from: "Prospects in mathematics", Princeton Univ. Press (1971) 77-169 MR0385006

[26] J Tits, Classification of algebraic semisimple groups, from: "Algebraic Groups and Discontinuous Subgroups", Amer. Math. Soc. (1966) 33-62 MR0224710

[27] J Tits, Reductive groups over local fields, from: "Automorphic forms, representations and L-functions, Part 1", (A Borel, W Casselman, editors), Proc. Sympos. Pure Math. 33, Amer. Math. Soc. (1979) 29-69 MR546588

[28] H C Wang, Topics on totally discontinuous groups, from: "Symmetric spaces", (W M Boothby, GL Weiss, editors), Dekker, New York (1972) 459-487 MR0414787

Department of Mathematics, Stanford University

Stanford, CA 94305, USA

vincent.emery@gmail.com

Received: 15 May 2013 